\title{
Coordinated Restraining of SSO via SSDC and SEDC in hybrid AC-DC System Containing MMC-HVDC
}

\author{
Li Shupeng ${ }^{1, a}$, Xu Zhenyu ${ }^{1, a}$, Qiao Yuchao ${ }^{1, b}$ \\ ${ }^{1}$ School of Electrical and Electronic Engineering, North China Electric Power University, \\ Beijing 102206, China

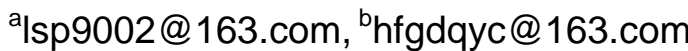

Keywords: MMC-HVDC; sub-synchronous oscillation; SSDC; SEDC; coordinated control strategy

\begin{abstract}
In this paper, aiming on the sub-synchronous oscillation (SSO) of hybrid AC-DC transmission system which contains modular multilevel converter based High Voltage Direct Current (MMC-HVDC), the coordinated control of sub-synchronous oscillation damping controller (SSDC) and supplementary excitation damping controller (SEDC) is researched. Firstly, aiming on the hybrid system, the structure and parameters of SSDC and SEDC are designed. Then, the practicability of the coordinated control of SSO via SSDC and SEDC is studied in both theory an electrical damping characteristics. Finally, the simulation model with 335 levels is built on PSCAD/EMTDC, and the results validate the correctness of the conclusion and the efficiency of the coordinated control strategy. It is found that SSDC and SEDC can superpose each other on increasing the electrical damping characteristic of whole system, and the coordinated control strategy proposed in this paper is practicabe.
\end{abstract}

\section{Introduction}

The sub-synchronous oscillation (SSO) is a serious stability problem of the grid which may cause the damage of the generator shaft. It occurred in the AC transmission system which contains series capacitor firstly [1]. Then, when the High Voltage Direct Current (HVDC) transmission line of SquareButte plant went into service, the SSO caused by HVDC was found [2]. Modular Multilevel Converter (MMC) presented by the German scholar R.Marquard is a new type of Voltage Source Converter (VSC) [3, 4]. Depending on its advantages of good expansibility, low harmonic distortion rate and little switching loss, the MMC based HVDC (MMC-HVDC) will develop into the main style of DC transmission in the future.

The MMC won't induce SSO as its output doesn't contain sub-synchronous component [5]. However, in the hybrid AC-DC system which contains series capacitor, there is a risk to occur SSO with the existing of the series capacitor [6].

The sub-synchronous oscillation damping controller (SSDC) and supplementary excitation damping controller (SEDC) are two effective measures to restrain SSO at present. The SSDC in MMC takes the sub-synchronous signal in the bus voltage as its input, and its output $P_{\mathrm{SSDC}}$ or $Q_{\mathrm{SSDC}}$ is added to the active power or reactive power reference value of the outer loop controller. The SEDC takes the speed information of the steam turbine high-pressure cylinder as the input signal, and its output is added to the excitation voltage. Although, the installation positions of SSDC and SEDC are different, both of them restrain SSO via changing the sub-synchronous torque relationship and enhancing the system's electrical damping.

A lot of research has been done to analyze the SSO in the hybrid AC-DC system containing VSC-HVDC. Reference [5][7,8] make a study of the sub-synchronous damping character of VSC-HVDC. The SSO of the system containing MMC-HVDC is analyzed in [6]. The SSDC which have single-channel or multi-channel structure is designed in [5][9], and these restrain effects are analyzed meanwhile. These researches only analyze the SSO and damping character of the hybrid AC-DC system when SSDC is used. Studies on the SSO of the system using SSDC and SEDC haven't been done yet. With the complexity of the grid and the increasing of the factor inducing SSO, 
these is a great necessary to have a research on the restraining strategy using SSDC and SEDC. In this paper, researches on the coordinated restraining of SSO via SSDC and SEDC in hybrid AC-DC system containing MMC-HVDC are done, and the coordinated control strategy is presented.

\section{The Hybrid AC-DC System to be Studied}

Firstly, the hybrid AC-DC transmission system containing MMC-HVDC should be clarified. And its structure is shown as Fig. 1.

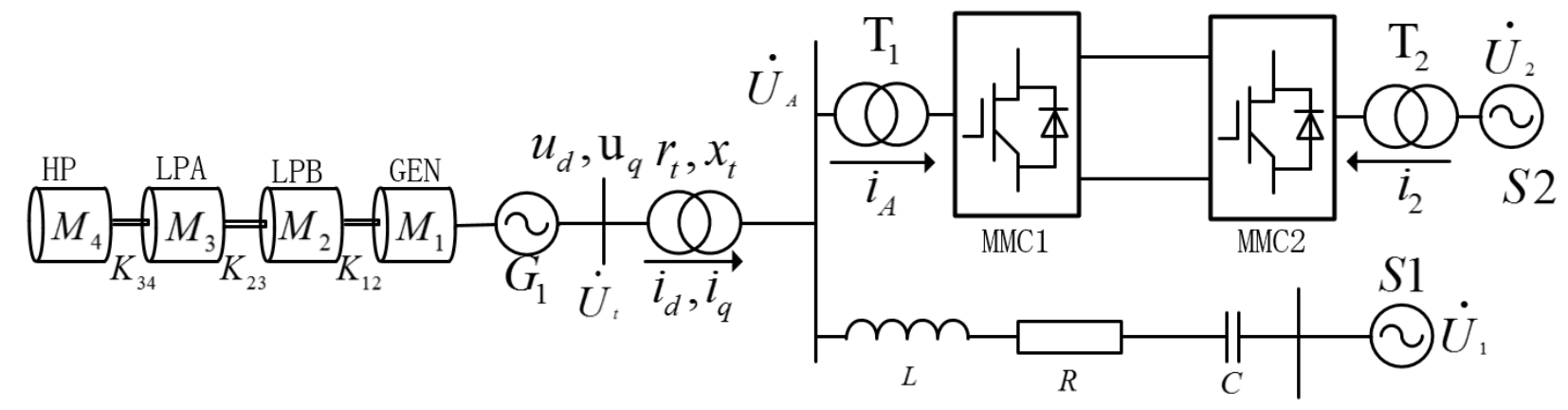

Fig. 1 Model of the hybrid AC-DC system

As it is shown in Fig. 1, $G_{1}$ is the studied generator while S1 and S2 are the equivalent AC system. HP, LPA, LPB and GEN indicate the four mass-blocks of the generator. The terminal voltage of generator is $U_{t}$ and its d-axis component is $u_{d}$ while q-axis component is $u_{q}$. $\mathrm{L}$ and $\mathrm{R}$ are the inductance and resistance of $\mathrm{AC}$ line and $\mathrm{C}$ indicates the series compensation capacitor. In the system studied in this paper, there are three sub-synchronous oscillation modalities, and they are $13.602 \mathrm{~Hz}$, $24.907 \mathrm{~Hz}$ and $29.517 \mathrm{~Hz}$.

\section{Design of the Damping Controller}

\section{A. Design of SSDC}

The SSDC studied in this paper adopts reactive power type whose output is added to the reference value of reactive power. The SSDC with this type can reduce the DC voltage fluctuation caused by the change of active power. Therefore, it benefits the stable operation of other converters[5].

The SSDC takes the sub-synchronous signal in the bus voltage as its input. After the measurement loop and phase lock loop (PLL), the speed signal $\Delta \omega$ can be got [10]. Then, $\Delta \omega$ is filtered and compensated in multiple channel and the output $Q_{\mathrm{SSDC}}$ is the addition of signals got from the three channels. The control signal $Q_{\mathrm{SSDC}}$ is added to the reactive power reference value of the outer loop controller. The structure of SSDC is shown as Fig. 2.

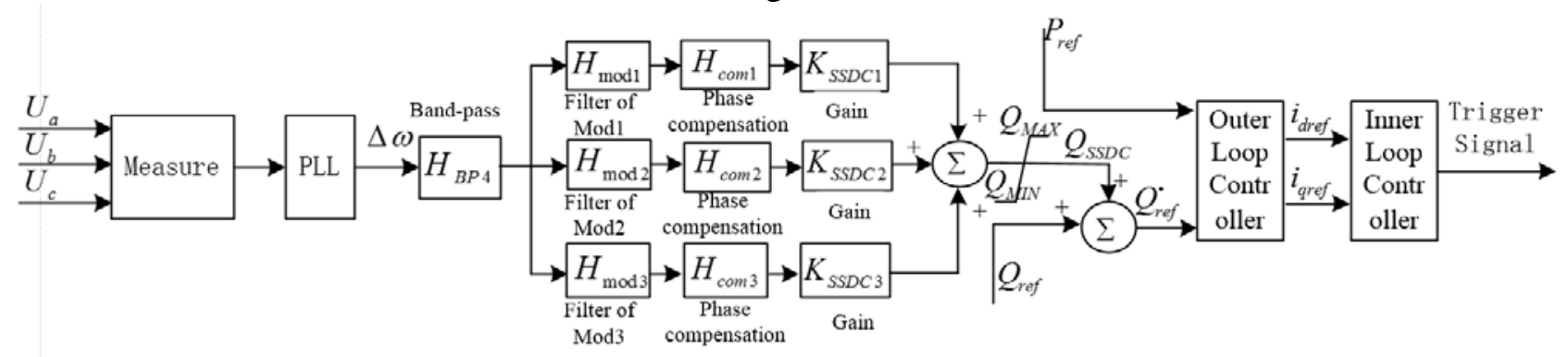

Fig. 2 Structure of SSDC with reactive power type

After the structure of SSDC is confirmed, the phase compensation and gain parameters should be calculated. And they can be obtained via test signal method discussed in references [11].Use the lead-lag link like $\left(1+s T_{1}\right) /\left(1+s T_{2}\right)$ to compensate the lagging phase of $\Delta Q$ to $\Delta \omega_{\text {mod }}$, and the time constant $T_{1}$ and $T_{2}$ can be calculated using equation (1). 


$$
a=T_{2} / T_{1}=(1-\sin \phi) /(1+\sin \phi), T_{1}=(2 \pi f \cdot \sqrt{a})^{-1}, T_{2}=a T_{1}
$$

In equation (1), $f$ is the modality frequency, and $\phi$ is the phase to be compensated. $T_{1}$ and $T_{2}$ are the time constant of phase compensation loop. The compensation phase, time constant and gain of the SSDC is shown as Tab. 1.

Tab. 1 Parameters of the SSDC

\begin{tabular}{ccccc}
\hline \multirow{2}{*}{ Frequency } & \multicolumn{2}{c}{ The compensation phase angle and time constant } & \multicolumn{2}{c}{ Gain } \\
\cline { 2 - 5 } & $\phi$ & $T_{1}$ & $T_{2}$ & $\mathrm{~K}$ \\
\hline Modality1:13.602 & 57.32 & 0.0197 & 0.0069 & 16.3 \\
Modality2:24.907 & 127.83 & 0.0276 & 0.0015 & 8.1 \\
Modality3:29.517 & 134.14 & 0.0266 & 0.0011 & 4.6 \\
\hline
\end{tabular}

\section{B. Design of SEDC}

The SEDC used in this paper is narrow-band [12], and its structure shown as Fig. 3 is multi-channel.

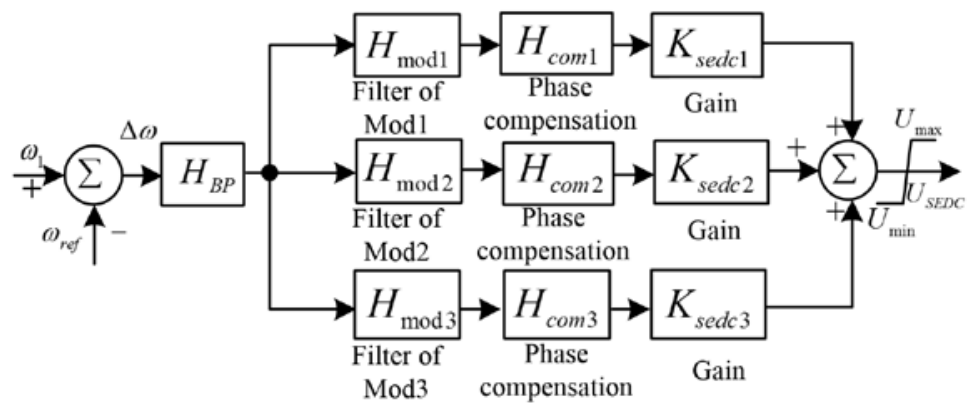

Fig. 3 Structure of SEDC

The phase compensation and gain parameters of SEDC also should be clarified when its structure is confirmed. And the method is the same as the SSDC. The compensation phase, time constant and gain of the SEDC is shown as Tab. 2.

Tab. 2 Parameters of the SEDC

\begin{tabular}{ccccc}
\hline \multirow{2}{*}{ Frequency } & \multicolumn{2}{c}{ The compensation phase angle and time constant } & \multicolumn{2}{c}{ Gain } \\
\cline { 2 - 5 } & $\phi$ & $T_{1}$ & $T_{2}$ & $\mathrm{~K}$ \\
\hline Modality1:13.602 & 42.49 & 0.0171 & 0.0080 & 20.8 \\
Modality2:24.907 & 82.02 & 0.0140 & 0.0029 & 28 \\
Modality3:29.517 & 68.85 & 0.0102 & 0.0028 & 37 \\
\hline
\end{tabular}

\section{Coordinated control strategy of SSDC and SEDC}

\section{A. Theory of the coordinated control strategy}

The restrain mechanism of SSDC and SEDC is different as the former's output is added to the outer loop controller of MMC and the latter's is added to the excitation device.

As an important part of the sub-synchronous torque relationship, the expression of electromagnetic torque $T_{\mathrm{e}}$ is shown as $T_{\mathrm{e}}=\psi_{\mathrm{d}} i_{\mathrm{q}}-\psi_{\mathrm{q}} i_{\mathrm{d}}$. Based on the generation's Park model, $T_{\mathrm{e}}$ can be simplified as $T_{\mathrm{e}} \approx\left[\left(X_{\mathrm{q}}-X_{\mathrm{d}}\right) i_{\mathrm{d}}+X_{\mathrm{ad}} i_{\mathrm{f}}\right] i_{\mathrm{q}}$. As $X_{\mathrm{q}} \approx X_{\mathrm{d}}$ and $\left(X_{\mathrm{q}}-X_{\mathrm{d}}\right) \ll X_{\mathrm{ad}}$ for the turbo-dynamo, $T_{\mathrm{e}}$ can be expressed as equation (2) finally.

$$
T_{\mathrm{e}} \approx X_{\text {ad }} i_{\mathrm{q}-\mathrm{f}-} i_{\mathrm{f}}+X_{\mathrm{ad}}\left(i_{\mathrm{q}-\mathrm{f} \sim} i_{\mathrm{f}}+i_{\mathrm{f}-} i_{\mathrm{q} \sim}\right)
$$

In equation (2), $i_{\mathrm{q}-}$ and $i_{\mathrm{q} \sim}$ indicate the power frequency and sub-synchronous frequency component of stator current. Analyzing equation (2), it can be found that $T_{\mathrm{e}}$ consists of two parts: the former $T_{\mathrm{e} 1}=X_{\mathrm{ad}} i_{\mathrm{q}-} i_{\mathrm{f}-\mathrm{a}}$ and the latter $T_{\mathrm{e} . \mathrm{sub}}=X_{\mathrm{ad}}\left(i_{\mathrm{q}-\mathrm{f}_{\mathfrak{f}}}+i_{\mathrm{f}-} i_{\mathrm{q} \sim}\right)$. 
The key to restrain SSO is control $T_{\text {e.sub }}$, and this object can be obtained by controlling the sub-synchronous frequency component of stator current and rotor current. Both SSDC and SEDC can produce additional electromagnetic torques, and they are expressed as $\Delta T_{\mathrm{SSDC}}$ and $\Delta T_{\mathrm{SEDC}} \cdot \Delta T_{\mathrm{e}}^{\prime}$ is the sum of $\Delta T_{\mathrm{e}}, \Delta T_{\mathrm{SSDC}}$ and $\Delta T_{\mathrm{SEDC}}$, and their relationship is shown as Fig.4. When the phase difference between $\Delta T_{\mathrm{e}}^{\prime}$ and $\Delta \omega$ can be guaranteed less than $90^{\circ}$, a positive electrical damping can be added to the system, and SSO restraining target can be got.

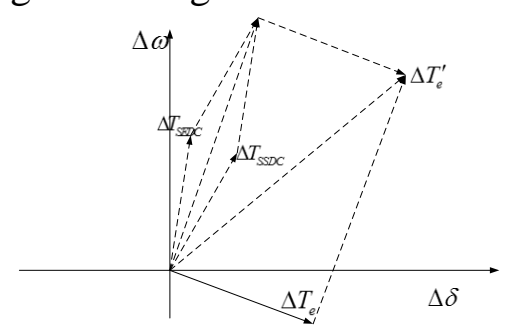

Fig. 4 Relationship of electromagnetic torques

When both SEDC and SSDC are used to restrain the SSO, they complement each other. As mentioned above, SSDC takes the speed signal as its input while SEDC takes the sub- synchronous signal in the bus voltage as its input. When large-disturbance occurs in the grid, there is enough synchronous signal in the bus voltage for SSDC to detect. The SSDC have a better restrain effect than SEDC at this moment [5]. But when small-disturbance happens in the grid, so little sub-synchronous signal exists in the bus voltage that it may be submerged by the noise. As a result of it, the SSDC cannot retrain the SSO effectively. Different from SSDC, the SEDC can detect this little sub-synchronous component easily as its input is the generator speed signal [3]. However, limited by the excitation system's capacity, SEDC has little effect on SSO when large-disturbance occurs.

\section{B. Analysis of the electrical damping characteristics}

As shown in Fig. 4, the synthesis of electromagnetic torque $\Delta T_{e}^{\prime}$ is the vector sum of $\Delta T_{e}, \Delta T_{S E D C}$ and $\Delta T_{S S D C}$. So the electrical damping produced by SSDC and SEDC should have a superposition relationship. Then, if the parameters of SSDC and SEDC are designed appropriately, they can be used in coordination to restrain SSO occurred in the hybrid system. The electrical damping of the system when using none of them, only using SSDC, only SEDC and using both of them are shown as Fig5.

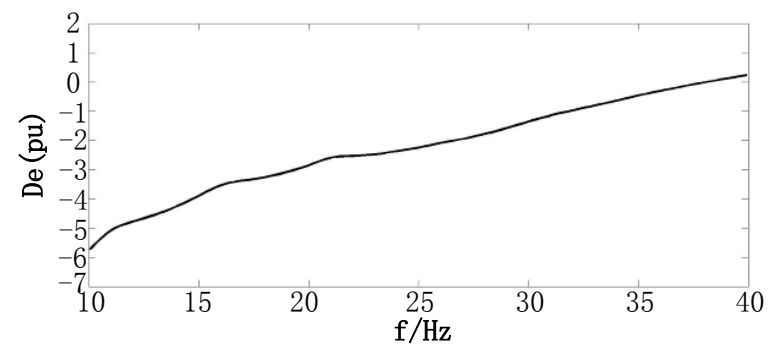

a) Electrical damping without SSDC and SEDC

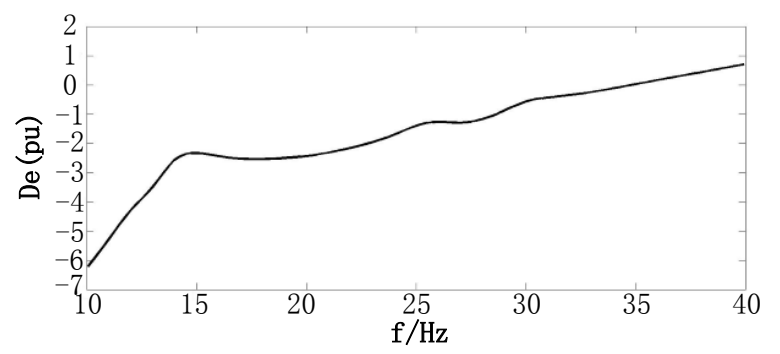

c) Electrical damping with SSDC

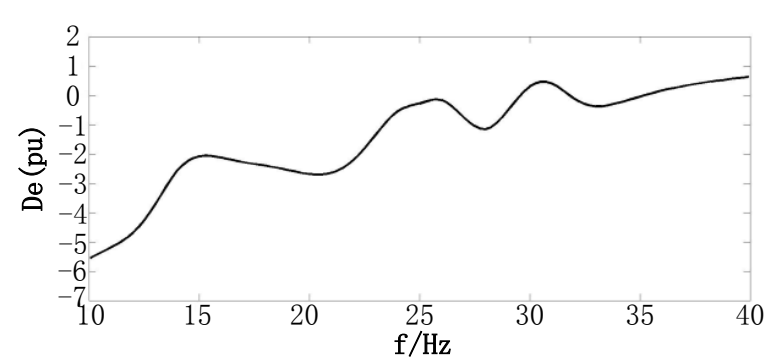

b) Electrical damping with SSDC

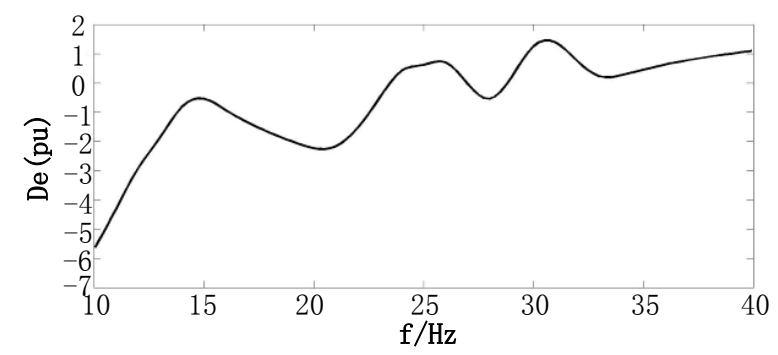

d) Electrical damping with SSDC and SEDC

Fig. 5 The electrical damping characteristics under all conditions

Analyzing Fig. 5, in can be found that before the addition of SSDC and SEDC, the SSO may occur as the electrical damping on three oscillation modalities are small. When SSDC or SEDC is used, the 
electrical damping can be strengthened to some extent, but it isn't strong enough. When SSDC and SEDC are used at the same time, the system's electrical damping is strengthened, and there is little possibility to incur SSO. Furthermore, the effects of SSDC and SEDC on strengthening electrical damping aren't obstruct each other, and have a superposition relationship. This conclusion is in correspondence with the theoretical analysis.

\section{Simulation and Verification}

In order to prove the efficiency of the coordinated control strategy, the simulation model with 335 levels shown as Fig. 1 is built on PSCAD/EMTDC. In this model, the rated voltage of the AC system is $500 \mathrm{kV}$. The rated voltage of T1and T2 are $22 \mathrm{kV} / 500 \mathrm{kV}$ and $500 \mathrm{kV} / 330 \mathrm{kV}$. The rated capacity of MMC-HVDC is $1000 \mathrm{MW}$ and the rated DC voltage is $\pm 350 \mathrm{kV}$. The outer control loop of MMC1 adopts constant DC voltage control and constant reactive power control while the outer control loop of MMC2 adopts constant active power control and constant reactive power control. The inner loop control of both adopt DQ decoupling control [13, 14].

When a single-phase grounding fault occurs on bus A, the dynamic changes of each modality speed under all conditions are shown as Fig. 6.

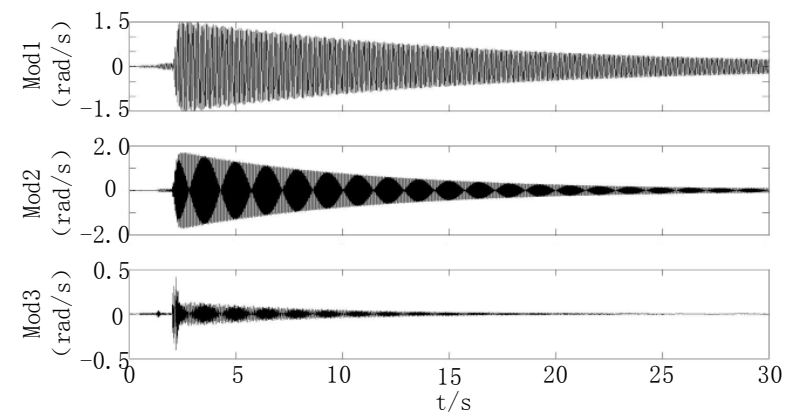

a) Modality speed without SSDC and SEDC

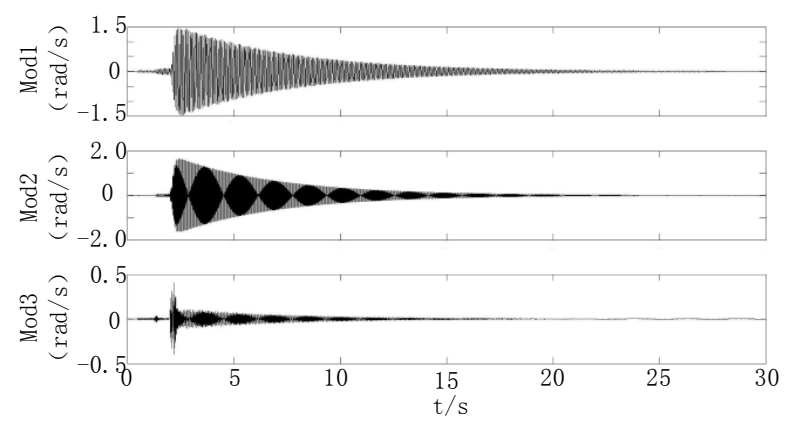

c) Modality speed with SEDC

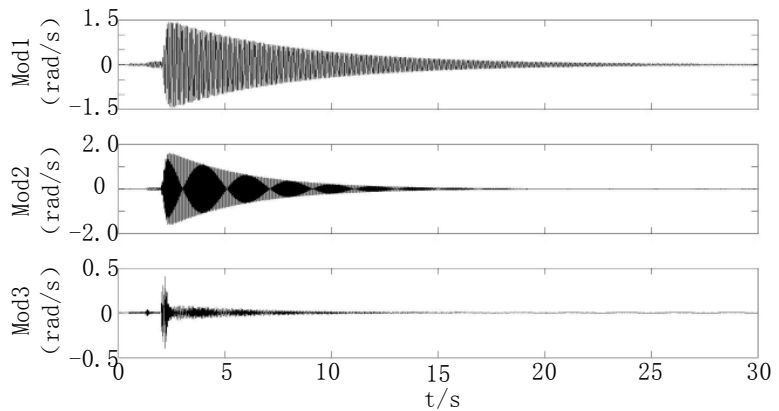

b) Modality speed with SSDC

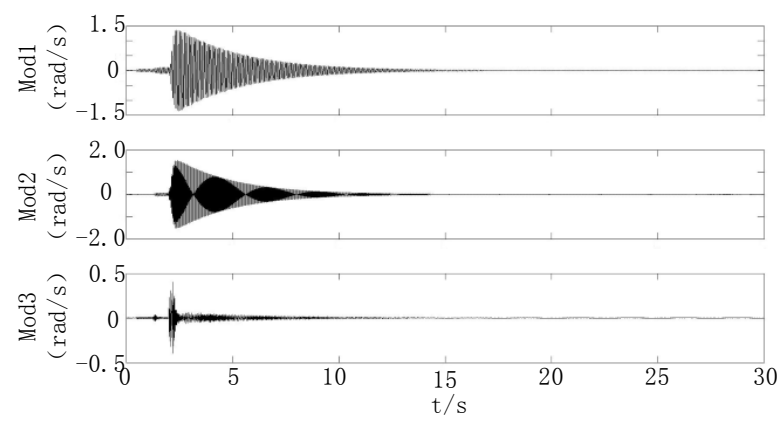

d) Modality speed with SSDC and SEDC

Fig. 6 The dynamic changes of each modality speed under all conditions

Analyzing Fig6 above, it can be found that before the addition of SSDC and SEDC the speed of Mod1 or Mod2 converge slowly. This condition may cause the tripping of protection device and even the damage of generator shaft. When SSDC or SEDC is added, the converge speed of Mod1 and Mod2 becomes faster. When SSDC and SEDC are added at the same time, the speed of Mod1 and Mod2 converges sharply. This phenomenon is consistent with the results got from electrical damping analysis.

\section{Summary}

In this paper, focusing on the SSO in hybrid AC-DC system containing MMC-HVDC, the coordinated restrain strategy of SSDC and SEDC is presented and its practicability is verified both in electrical damping analysis and simulation. The conclusion is summarized as:

(1) The restrain strategy of using SSDC and SEDC at the same time is practicable. 
(2) The effects of SSDC and SEDC on strengthening electrical damping aren't obstruct each other, and have a superposition relationship.

(3) When adopting the strategy of using SSDC and SEDC at the same time, the sub-synchronous signal motivated by fault can be restrained quickly and the risk of occurring SSO reduces quickly.

\section{References}

[1] Hall M.C., Daniels R.L., Ramey D.G., A new technique for sub-synchronous resonance analysis and an application to the kaiparowits system[J]. IEEE Power Apparatus and Systems, 1977, 96(4):1251-1255.

[2] Li, Z., Wan, Q.L., Zhang, X.P., STUDY ON THE SSO CAUSED BY HVDC LINK IN HYBRID AC-DC POWER SYSTEM[C], IET Proceedings on AC and DC Power Transmission, 2010:1-5.

[3] Wei Yanfang, Wei Zhinong, Sun Guoqiang.New HVDC power transmission technology: MMC-HVDC[J].Electric Power Automation Equipment, 2012, 32(7) :1-9.

[4] Zuniga-Garcia, L. , Zaragoza-Hernandez, A. , Nadal-Martinez, J.M.. MMC technology and its participation in the integration of variable renewable energies and implementation of DC grids[C]// 2014 IEEE International Autumn Meeting on Power, Electronics and Computing. Ixtapa: IEEE, 2014: 1-6.

[5] Zheng Chao, Tang Yong, Ma Shiying, et al . Subsynchronous Oscillation Damping Characteristic Analysis for VSC-HVDC Based on Its Equivalent Simulation Model [J].Proceedings of the CSEE, 2007, 27(31):33-39.

[6] Li Haifeng.The Analysis and Elimination of Subsynchronous Oscillation for AC-DC Hybrid System[D].Beijing:North China Electric Power University, 2013.

[7] Xu Kun.Research on Subsynchronous Damping Effect of VSC-HVDC[D].Beijing:North China Electric Power University, 2012.

[8] Gao Benfeng, Xu Kun, Xiao Xiangning, et al.Subsynchronous Oscillation Caused by VSC-HVDC for Damping Fixed Series Compensation[J].East China Electric Power, 2011, 39(4):556-560.

[9] Jiang Ping, Hu Tao, Wu Xi.Multi-channel supplementary damping control in VSC-HVDC for SSO suppression [J].Electric Power Automation Equipment, 2011, 31(9):27-31.

[10]Gao Benfeng, Zhao Chengyong, Xiao Xiangning, et al. Design and Implementation of SSDC for HVDC[J].High Voltage Engineering, 2010, 36(2):501-506.

[11]Zheng Xu, Zhouyan Feng, A Novel Unified Approach For Analyzing Small-Signal Stability of Power System [C]//IEEE PES Winter Meeting. IEEE, 2000:963-967.

[12]Zhang Fan, Xu Zheng. Studies on a Supplementary Excitation Damping Controller for Mitigating SSR [J].Automation of Electric Power System, 2007,31(23):24-29.

[13]Guan Minyuan, Xu Zheng.Modeling and Control of ModularMultilevel Converter in HVDC Transmission [J].Automation of Electric Power System, 2010, 34(19):64-68.

[14] Zhao Yan, Hu Xuehao, Tang Guangfu, et al. A study on MMC model and its current control strategies [C]// 2nd IEEE International Symposium on Power Electronic Generation System. Hefei: IEEE, 2010: 259 - 264 